\title{
Aortic valve reconstruction using autologous pericardium for patients aged less than 60 years
}

Shigeyuki Ozaki, MD, PhD, Isamu Kawase, MD, PhD, Hiromasa Yamashita, MD, Yukinari Nozawa, MD, Mikio Takatoh, MD, So Hagiwara, MD, and Nagaki Kiyohara, MD

Objective: We have performed an original aortic valve reconstruction using autologous pericardium. The feasibility for patients aged less than 60 years is reviewed.

\begin{abstract}
Methods: From April 2007 to April 2013, aortic valve reconstruction was performed in 108 patients aged less than 60 years. A total of 51 patients had aortic stenosis, 7 patients had annuloaortic ectasia, 7 patients had infective endocarditis, and 43 patients had aortic regurgitation. Fifty-seven patients had bicuspid valves, and 11 patients had unicuspid valves. There were 75 male and 33 female patients, with a mean age of $47.8 \pm 11.2$ years. Preoperative echocardiography showed an average peak pressure gradient of $86.1 \pm 35.1 \mathrm{~mm} \mathrm{Hg}$ with aortic stenosis. The surgical procedure is based on the independent tricuspid replacement using autologous pericardium. First, the distance between the commissures is measured using an original sizing apparatus, and then the pericardial cusp is trimmed using an original template and sutured to the annulus.

Results: There was no conversion to prosthetic valve replacement. There were no in-hospital mortalities. Postoperative echocardiography showed an average peak pressure gradient of $14.8 \pm 7.8 \mathrm{~mm} \mathrm{Hg} 1$ week after surgery and $12.8 \pm 3.1 \mathrm{~mm} \mathrm{Hg} 4$ years after surgery. One patient required reoperation because of infective endocarditis. The other 107 patients showed less than mild aortic regurgitation. No thromboembolic events were recorded. The mean follow-up period was $34.2 \pm 15.7$ months. Freedom from reoperation was $98.9 \%$ with 76 months of follow-up.
\end{abstract}

Conclusions: Original aortic valve reconstruction was feasible for patients aged less than 60 years. Long-term data will be disclosed in the future. (J Thorac Cardiovasc Surg 2014;148:934-8)

Aortic valve reconstruction is the gold standard of surgical treatment for various aortic valve diseases, especially for adults. Bioprosthetic valves are used increasingly because of the complications with inevitable postoperative anticoagulation of mechanical valves. ${ }^{1,2}$ However, despite the progress of the design and construction of prosthetic valves, hemodynamic performance is not yet comparable to that of native aortic valves. Moreover, replacement with a bioprosthesis can cause accelerated degeneration in young patients because of fibrosis and calcification. ${ }^{3}$ Immune reaction may play a role in the degeneration of the implanted bioprosthesis, especially in younger patients. ${ }^{4,5}$

Aortic valve repair, the Ross operation, and balloon aortic valvuloplasty are the other surgical treatment options

From the Department of Cardiovascular Surgery, Toho University Ohashi Medical Center, Tokyo, Japan.

Disclosures: Authors have nothing to disclose with regard to commercial support.

Read at the 94th Annual Meeting of The American Association for Thoracic Surgery, Toronto, Ontario, Canada, April 26-30, 2014.

Received for publication April 9, 2014; revisions received May 10, 2014; accepted for publication May 16, 2014; available ahead of print June 20, 2014.

Address for reprints: Shigeyuki Ozaki, MD, PhD, Department of Cardiovascular Surgery, Toho University Ohashi Medical Center, 2-17-6 Ohashi, Meguro, Tokyo, 153-8515 Japan (E-mail: ozakis@oha.toho-u.ac.jp).

$0022-5223 / \$ 36.00$

Copyright (c) 2014 by The American Association for Thoracic Surgery

http://dx.doi.org/10.1016/j.jtcvs.2014.05.041 for aortic valve disease, but they have been performed in only a select group of patients by a limited group of well-experienced surgeons or interventionists.

Our original aortic valve reconstruction has been performed since April 2007. The initial report of 88 patients undergoing this operation was published in $2011 .^{6}$ This is a unique operation that consists of independent replacement of 3 aortic valve cusps by glutaraldehyde-treated autologous pericardium and that preserves the natural motion of the aortic annulus during the cardiac cycle. In the current study, we inspected the feasibility of our original aortic valve reconstruction for patients aged less than 60 years.

\section{METHODS}

Our original aortic valve reconstruction and the clinical study of this operation were approved by the institutional review board of Toho University Ohashi Medical Center. All patients underwent this operation after written, informed consent had been obtained.

Our original aortic valve reconstruction was performed between April 2007 and April 2013 in 108 patients aged less than 60 years. We retrospectively reviewed our clinical data of all 108 patients and evaluated their short- and midterm results.

There were 75 male and 33 female patients, with a mean age of $47.8 \pm 11.2$ years. The age distribution of all 108 patients of the current study group is shown in Figure 1. A total of 51 patients had aortic stenosis (AS), 43 patients had aortic regurgitation, 7 patients had annuloaortic ectasia, and 7 patients had infective endocarditis. A total of 57 patients had bicuspid aortic valves, and 11 patients had unicuspid valves. 


\section{Abbreviation and Acronym}

$\mathrm{AS}=$ aortic stenosis

Preoperative echocardiography showed that the peak pressure gradient through the aortic valve averaged $86.1 \pm 35.1 \mathrm{~mm} \mathrm{Hg}$ in 51 patients with AS.

\section{Surgical Technique}

The surgical technique of our original aortic valve reconstruction has been reported. ${ }^{6,7}$ Preparation of the autologous pericardium began with cleansing fat and other redundant tissue on the outer surface of the pericardium using the Harmonic scalpel (Ethicon Endo-Surgery, Inc, Cincinnati, Ohio). The pericardium was excised to approximately $7 \times 8 \mathrm{~cm}$ and treated with $0.6 \%$ glutaraldehyde solution with buffer for 10 minutes. The treated pericardium was rinsed 3 times using a sterilized physiologic saline solution, with a duration of 6 minutes per rinse. All of the aortic valve reconstructive procedures were performed during cardioplegic arrest on cardiopulmonary bypass.

First in the procedure, the diseased cusps are excised meticulously. In the case of thick calcification along the aortic annulus, the Cavitron Ultrasonic Surgical Aspirator (SonoSurg, Olympus, Tokyo, Japan) is helpful in removing it without damaging annular tissue. The distance between each commissure is measured using our original sizing apparatus. From the glutaraldehyde-treated autologous pericardium, the new cusp with the size corresponding to the measured value is trimmed using our original template. Last, the annular margin of the pericardial cusp is sutured with running 4-0 monofilament stitches to each annulus. The running suture should be performed to make a nice gather, creating a 3-dimensional natural bulge of the cusps. Each pericardial cusp is sewn all the way to the top of the commissure. The reason behind this design is to have a longer coaptation zone, up to the same horizontal plane as the commissures. Commissural coaptation is secured with additional 4-0 monofilament sutures. The coaptation of the 3 new cusps is always ensured with direct vision under negative pressure made by a left ventricular vent before aortotomy closure. In patients with aortic regurgitation or bicuspid/unicuspid valves, we encircle the aorta at the commissural level with a 5-mm-wide felt strip after de-clamping the aorta to prevent the future dilatation of the aorta and eventual recurrence of regurgitation (Figure 2).

\section{Follow-up}

All patients were followed up at the outpatient clinic of Toho University Ohashi Medical Center or at the referral hospital. Echocardiographic evaluation was performed 1 week, 1 month, 3 months, and every 6 months after surgeries.

\section{Statistical Analysis}

Data are presented as mean \pm standard deviation. Survival and freedom from reoperation rate were calculated by the Kaplan-Meier method.

\section{RESULTS}

There were no conversions to prosthetic valve replacement. There were no in-hospital mortalities recorded. Postoperative echocardiography showed an average peak pressure gradient of $14.8 \pm 7.8 \mathrm{~mm} \mathrm{Hg} 1$ week after surgery and $12.8 \pm 3.1 \mathrm{~mm} \mathrm{Hg} 4$ years after surgery. No cusp calcification was recorded during the entire follow-up period. One patient required reoperation because of infective endocarditis. The other 107 patients showed less than mild aortic regurgitation. No thromboembolic events were recorded even without anticoagulation. The mean followup period was $34.2 \pm 15.7$ months. Survival and freedom from reoperation were $96.9 \%$ and $98.9 \%$, respectively, at 76 months follow-up (Figure 3).

Because our technique is tricuspid reconstruction for all patients, including those with bicuspid or tricuspid aortic valves, we held the comparative study in subgroups of patients who originally had tricuspid, bicuspid, or unicuspid aortic valves. Furthermore, the transition of averaged peak pressure gradient through the aortic valves showed no significant differences in the 3 subgroups (Figure 4).

\section{DISCUSSION}

The choice of strategy among variable aortic valve surgical procedures is still controversial, especially for younger patients. The available surgical treatments of aortic valve disease are repair and replacement of the aortic valve. Valve-sparing operations can be categorized broadly into interventions on the aortic valve itself and those aimed at repairing the proximal aorta.

Aortic valve reconstruction is now acknowledged as a safe and effective operation for aortic valve disease. The recent large-scale study of aortic valve reconstruction with 7883 Japanese patients from 2005 to 2008 showed an operative mortality of $3 \%{ }^{8}$ Conventional aortic valve reconstruction presents complications resulting from prosthetic valves. Mechanical valves require lifelong anticoagulation, and bioprosthesis is associated with high rates of structural valvular degeneration. ${ }^{9,10}$ The stented bioprosthesis showed progressive calcification at the base of each cusp, notably near the commissure. ${ }^{11}$ Even with a stentless prosthesis, calcification may be present along the aortic wall. ${ }^{11}$ During the follow-up of all 108 patients aged less than 60 years in the current series, we found no signs of calcification. The following case is a good example. We performed our original aortic valve reconstruction on a 14-year-old boy with a failed unicuspid aortic valve (Figure 5, A). Echocardiographic follow-up 3.5 years

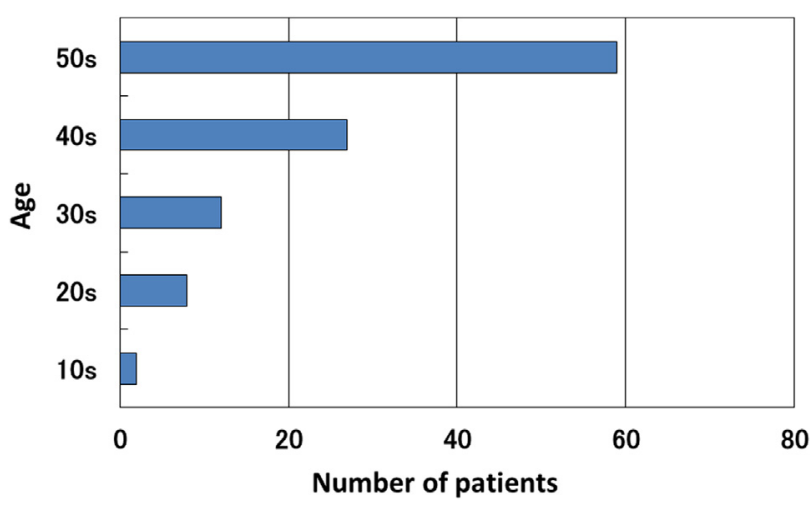

FIGURE 1. Age distribution of all 108 patients in the current study. 


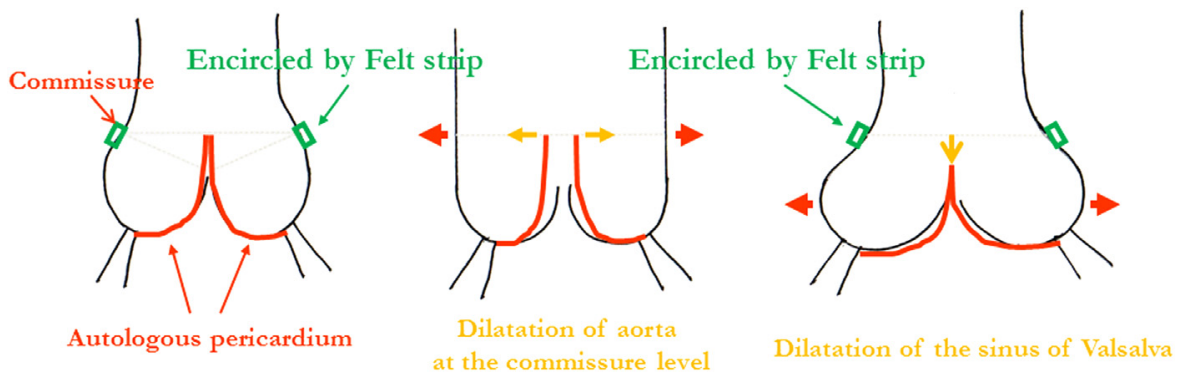

FIGURE 2. Mechanism and effect of the encircling felt strip at the commissural level.

after operation showed no signs of calcification and natural motion of the 3 cusps (Figure 5, B). Moreover, patients aged less than 40 years showed excellent hemodynamics after surgery, including this 14-year-old boy (Figure 5, C).

The Ross procedure was developed in an attempt to provide a permanent biological aortic valve prosthesis using the autologous pulmonic valve. This operation is especially effective for pediatric patients. It provides excellent hemodynamics but requires expertise and has several disadvantages, among which are failure of the pulmonary homograft and dilatation of native aortic root or pulmonary autograft. ${ }^{12,13}$ In the current study, because of the felt strip encircling the aorta at the commissural level and the running suture of the 3 cusps, we observed no postoperative annular dilation even in the

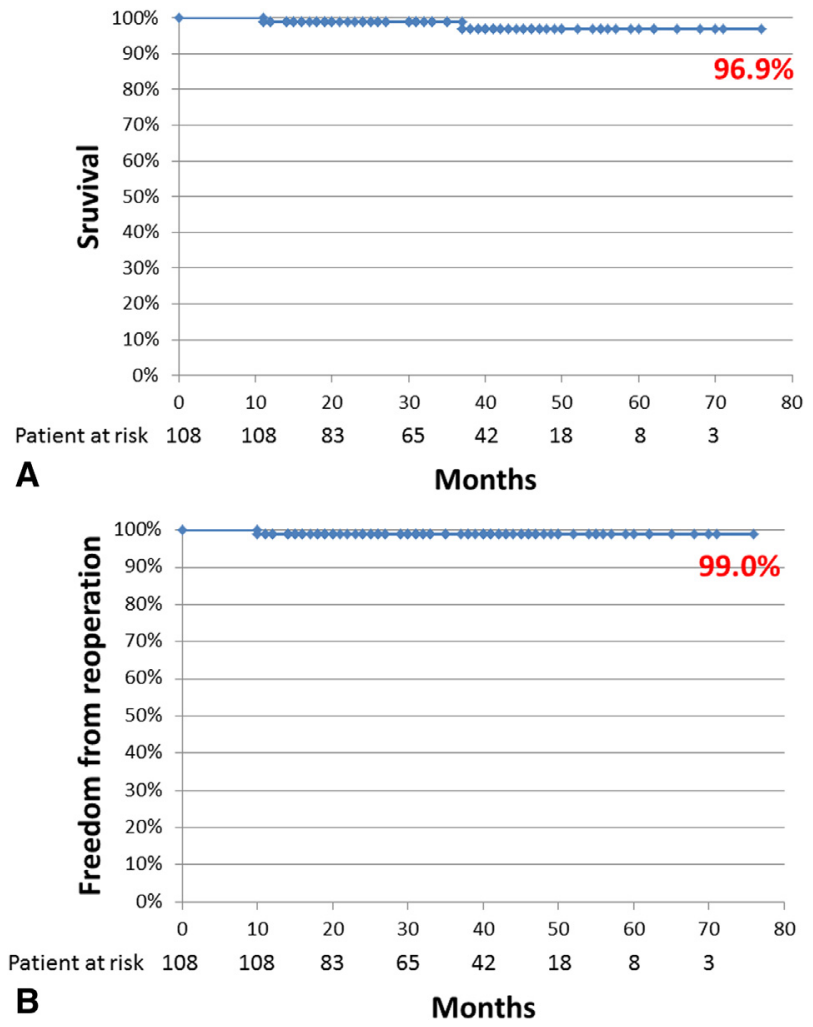

FIGURE 3. A, Survival curve after aortic valve reconstruction of 108 patients aged less than 60 years. B, Freedom from reoperation curve after aortic valve reconstruction of 108 patients aged less than 60 years. 14-year-old boy. Both of these procedures prevent the dilation of the aorta and aortic annulus, which in turn prevents future aortic regurgitation (Figure 5, D).

Various aortic valve repair techniques have been reported showing good results. ${ }^{14-19}$ Successful bicuspidization repair also has been reported. ${ }^{19}$ On the contrary, with the congenital bicuspid aortic valve, AS might occur at a mean age less than 60 years, and aortic regurgitation might develop at a mean age less than 30 years. ${ }^{20,21}$ Although the exact mechanism of proximal aortic dilatation has not been clarified, a report shows that the significantly higher shear forces might have an impact on the development of aortic dilatation in patients with bicuspid aortic valves. ${ }^{22}$ Original aortic valve reconstruction is exclusively tricuspidization, which is basically a tricuspid replacement using autologous pericardium. We believe tricuspidization is the ideal reconstructive technique rather than bicuspidization. The orientation of the cusps in a tricuspid valve allows each cusp to fully open while maintaining the circular shape of the aortic valve, which is why we perform our original

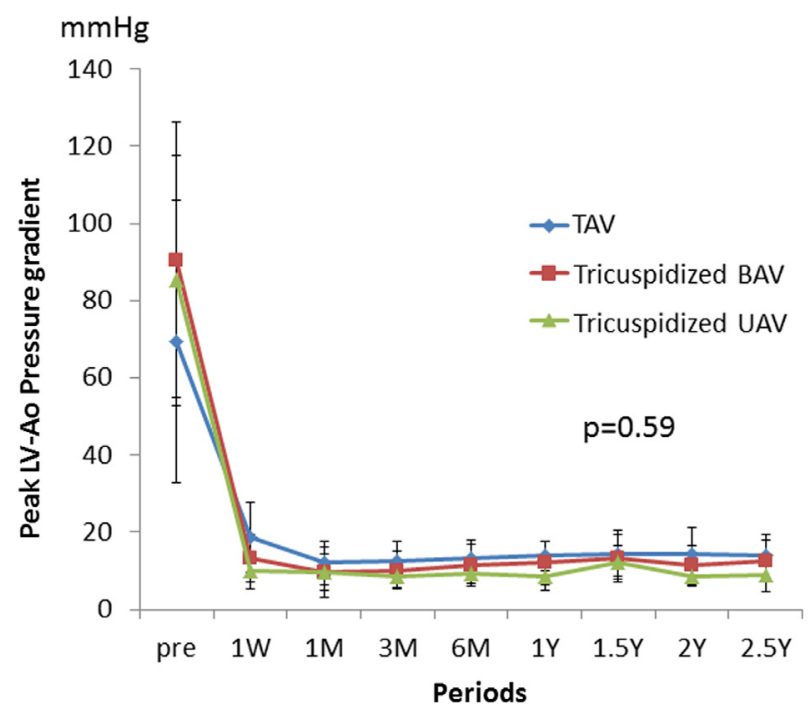

FIGURE 4. Transition of averaged peak pressure gradient through the aortic valve from preoperative data to postoperative follow-up: Comparative study among 3 patients' subgroups of preoperative tricuspid, bicuspid, and unicuspid aortic valves. $B A V$, Bicuspid aortic valve; $L V$-Ao, left ventricular-aortic; $T A V$, tricuspid aortic valve; $U A V$, unicuspid aortic valve. 


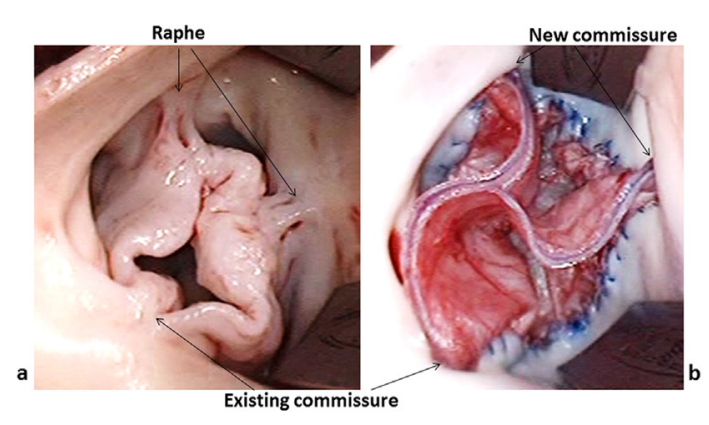

A

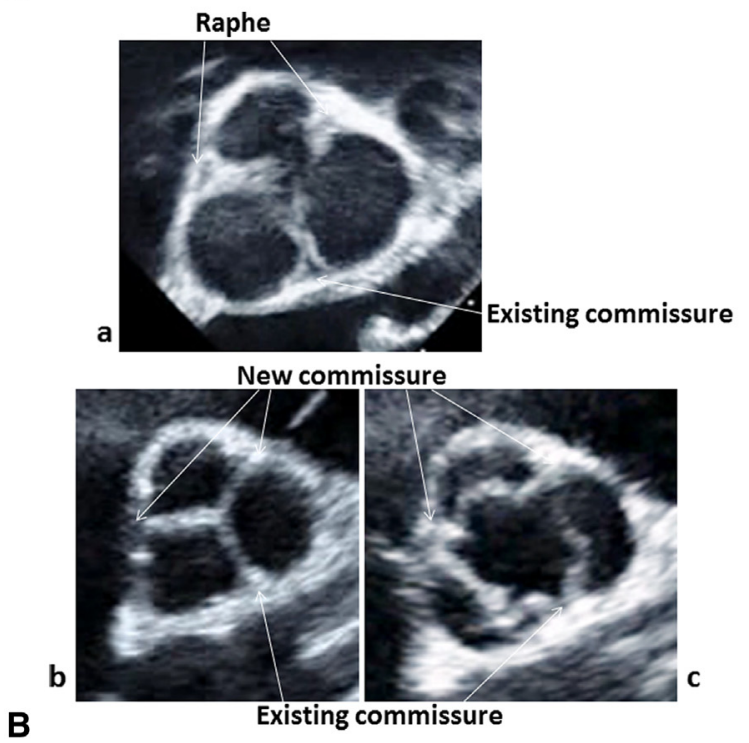

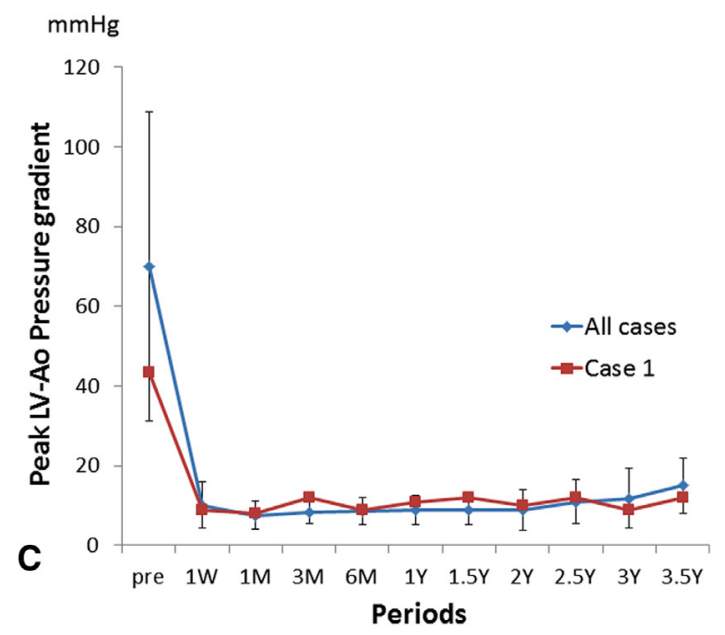

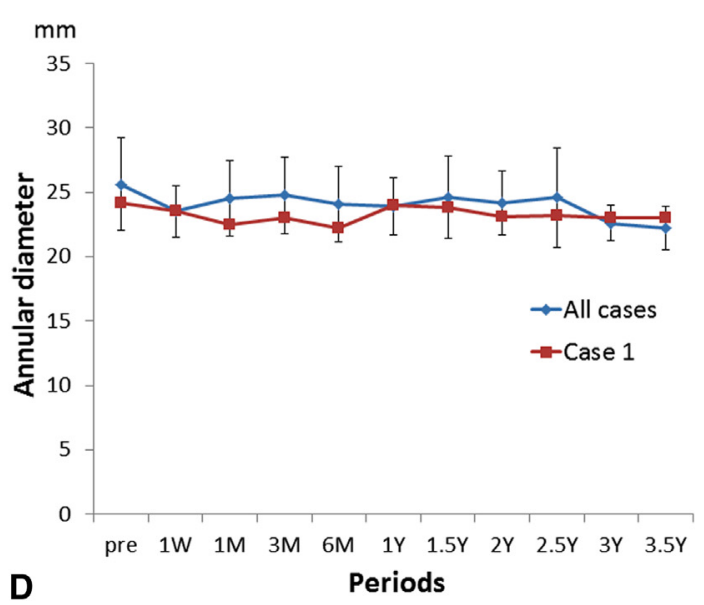

FIGURE 5. A, Operative view of pre- (a) and post- (b) tricuspidized repair of unicuspid aortic valve in a 14-year-old boy. B, Preoperative echocardiography of unicuspid aortic valve in a 14-year-old boy (a) and diastolic and systolic phases of tricuspidized valve 3.5 years after operation (b, c). C, Average peak pressure gradient through the aortic valve in patients aged less than 40 years (red line indicates transition of peak pressure gradient in a 14-year-old boy with congenital unicuspid valve). D, Average aortic valve annular diameter in patients aged less than 40 years (red line indicates transition of aortic valve annular diameter in a 14-year-old boy with congenital unicuspid valve). $L V$-Ao, Left ventricular-aortic.

aortic valve reconstruction for tricuspidization. In this study, we observed the compatible hemodynamics of tricuspidized bicuspid or unicuspid aortic valves with tricuspid valve cases (Figure 4). Tricuspidization is important for full opening of the cusps.

This procedure preserves the coordination of the left ventricle, aortic valve annulus, aortic valve cusps, sinus of Valsalva, and sinotubular junction to maximize aortic valve function. Natural annular motion can be preserved with our original aortic valve reconstruction and contributes to the postoperative low pressure gradient through the pericardial aortic valve.

\section{Study Limitations}

A limitation of the current study is the lack of long-term follow-up. We will continue to monitor the results for a longer follow-up period with a larger number of patients.

\section{CONCLUSIONS}

Our original aortic valve reconstruction with glutaraldehyde-treated autologous pericardium has shown good short- and midterm results for patients aged less than 60 years. Our results show that tricuspidization not only preserves the natural motion of the aortic valve but also maintains a low pressure gradient and minimal regurgitation. Long-term data will be disclosed in the future.

\section{References}

1. North RA, Sadler L, Stewart AW, McCowan LM, Kerr AR, White HD. Long-term survival and valve-related complications in young women with cardiac valve replacements. Circulation. 1999;99:2669-76.

2. Edmunds LH. Thrombotic and bleeding complications of prosthetic heart valves. Ann Thorac Surg. 1987;44:430-45.

3. Khan SS, Trento A, DeRobertis M, Kass RM, Sandhu M, Czer LS, et al. Twenty-year comparison of tissue and mechanical valve replacement. J Thorac Cardiovasc Surg. 2001;122:257-69. 
4. Dahm M, Husmann M, Eckhard-Mayer, Prufer D, Groh E, Oelert H. Relevance of immunologic reactions for tissue failure of bioprosthetic heart valves. Ann Thorac Surg. 1995;60:S348-52.

5. Human P, Zilla P. Characterization of the immune response to valve bioprostheses and its role in primary tissue failure. Ann Thorac Surg. 2001;71: S385-8.

6. Ozaki S, Kawase I, Yamashita H, Uchida S, Nozawa Y, Matsuyama T, et al. Aortic valve reconstruction using self-developed aortic valve plasty system in aortic valve disease. Interact Cardiovasc Thorac Surg. 2011;12:550-3.

7. Ozaki S, Kawase I, Yamashita H, Uchida S, Nozawa Y, Takatoh M, et al. A total of 404 cases of aortic valve reconstruction with glutaraldehyde-treated autologous pericardium. J Thorac Cardiovasc Surg. 2014;147:301-6.

8. Handa N, Miyata H, Motomura N, Nishina T, Takamoto S. Procedure- and age-specific risk stratification of single aortic valve replacement in elderly patients based on Japan adult cardiovascular surgery database. Circ J. 2012; 76:356-64.

9. Jamieson WRE, Burr LH, Miyagishima RT, Germann E, Macnab JS, Stanford E, et al. Carpentier-Edwards supra-annular aortic porcine bioprosthesis: clinical performance over 20 years. J Thorac Cardiovasc Surg. 2005;130: 994-1000.

10. Salem DN, Stein PD, Al-Ahmad A, Bussey HI, Horstkotte D, Miller N, et al. Antithrombotic therapy in valvular heart disease - native and prosthetic. Chest. 2004; 126:457S-82S.

11. Ozaki S. Pathophysiology of Calcification of Bioprosthetic Heart Valves: An Experimental Investigation. Acta Biomedica Lovaniensia. Vol. 238. Leuven, Belgium: Leuven University Press; 2001.

12. Vahanian A, Alfieri O, Andreotti F, Antunes MJ, Baron-Esquivias G, Baumgartner H, et al. Guidelines on the management of valvular heart disease (version 2012). Eur Heart J. 2012;33:2451-96.
13. Nishimura RA, Otto CM, Bonow RO, Ruiz CE, Carabello BA, Skubas NJ, et al 2014 AHA/ACC Guideline for the Management of Patients with Valvular Heart Disease. J Am Coll Cardiol. 2014;63:e57-185.

14. Minakata K, Schaff HV, Zehr KJ, Dearani JA, Daly RC, Orszulak TA, et al Is repair of aortic valve regurgitation a safe alternative to valve replacement? J Thorac Cardiovasc Surg. 2004;127:645-53.

15. Rao V, Van Arsdell GS, David TE, Azakie A, Williams WG. Aortic valve repair for adult congenital heart disease: a 22-year experience. Circulation. 2000; 102(Suppl):III-40-3.

16. Veldman GR, Connolly HM, Orszulak TA, Dearani JA, Schaff HV. Fate of bicuspid aortic valves in patients undergoing aortic root repair or replacement for aortic root enlargement. Mayo Clin Proc. 2006;81:322-6.

17. Casselman FP, Gillinov AM, Akhrass R, Kasirajan V, Blackstone EH, Cosgrove DM. Intermediate-term durability of bicuspid aortic valve repair for prolapsing leaflet. Eur J Cardiothorac Surg. 1999;15:302-8.

18. Tolan MJ, Daubeney PE, Slavik Z, Keeton BR, Salmon AP, Monro JL. Aortic valve repair of congenital stenosis with bovine pericardium. Ann Thorac Surg. 1997;63:465-9.

19. Doss M, Moid R, Wood JP, Miskovik A, Martens S, Moritz A. Pericardial patch augmentation for reconstruction of incompetent bicuspid aortic valves. Ann Thorac Surg. 2005;80:304-7.

20. Roberts WC. Congenitally bicuspid aortic valve - a study of 85 autopsy cases. Am J Cardiol. 1970;26:72-83.

21. Olson LJ, Subramanian R, Edwards WD. Surgical pathology of pure aortic-insufficiency - a study of 225 cases. Mayo Clin Proc. 1984;59:835-41.

22. Meierhofer C, Schneider EP, Lyko C, Hutter A, Martinoff S, Markl M, et al. Wall shear stress and flow patterns in the ascending aorta in patients with bicuspid aortic valves differ significantly from tricuspid aortic valves: a prospective study. Eur Heart J Cardiovasc Imaging. 2013;14:797-804. 